

\title{
ENERGY ABSORPTION OF A HELICOIDAL BISTABLE STRUCTURE
}

\author{
SeubPong LeElavanichKul, ANDREJ CherkaeV, \\ DANIEL O. ADAMS AND FLORIAN SOLZBACHER
}

\begin{abstract}
The concept of a bistable structure as an energy absorbing structure is presented. A bistable structure is capable of distributing damage throughout the whole body, thus increasing the amount of energy dissipation. Using this concept, a helicoidal bistable structure is developed. We investigate a structure in which a helicoidal orthotropic shell envelops a solid cylinder. The center core of the structure is treated as a linear-elastic isotropic material. Numerical simulations of damage (breakages) are performed under static loads, and the force-displacement relation is obtained as a result. As an energy absorbing structure, the helicoidal bistable structure can absorb more energy than a conventional structure before it fails. The helicoidal bistable structure can be designed to sustain a small elongation while still being able to release energy effectively. The additional twisting degrees of freedom correspond to the additional energy released in the process of elongation.
\end{abstract}

\section{Introduction}

The total fracture energy is an important consideration when determining the ability of a structure to withstand dynamic loading. In most structures, however, localized yielding occurs at a particular location such that the total energy associated with a fracture in the structure is relatively small.

Bistable structures allow an increase in the total fracture energy. In a bistable structure there are redundant localized load paths, so that when one load path yields and subsequently fails, the remaining load paths can carry the load, preserving structural integrity. A bistable structure can be developed by forming a chain of bistable structural elements such that multiple regions of the structure may exhibit yielding behavior prior to ultimate structural failure. In other words, yielding is delocalized, occurring in a larger fraction of the structure.

The analytical solution to the dynamic loading of a chain of bistable structural elements was first proposed by Slepyan and Troyankina [1984; 1988]. This dynamic yielding model was later extended by Balk et al. [2001a; 2001b] to the case of reversible two-phase chains of bistable structural elements. The dynamic response of elastic-brittle bistable structures was also studied by Cherkaev et al. [2005; 2005]. The response of chains of bistable structural elements under quasistatic and dynamic loading was investigated by Cherkaev and Zhornitskaya [2003]. The numerical results obtained showed that a chain of bistable links can withstand impact energy levels that are several times larger than for a conventional chain without bistable behavior.

A special structural design using a "waiting element" [Cherkaev and Slepyan 1995] has been used to increase stability. Waiting elements are parts of the structure that are initially not active and which remain nonactive until the strain becomes sufficiently large. Each link in the chain transitions from the lower

Keywords: bistable structure, helicoidal composites, energy absorption, damage. 
strain state to the higher strain state, which produces a transition wave that propagates along the chain. Unlike conventional structures, in which the strain is concentrated near the impact zone, a structure with waiting elements distributes the strain over a larger area. Cherkaev et al. [2006] showed that by using an elastic-plastic material in the redundant load path, or waiting element, the cross sectional area of this portion could be made significantly smaller than the main load path, or main element, because an elasticplastic material can sustain larger deformations beyond the material yield strength. An elastic-brittle material, on the other hand, fails once the yield strength is reached. This concept was also explored experimentally by Whitman and La Saponara [2007a; 2007b].

The bistable structure presented in our previous work [Cherkaev et al. 2006] (see Figure 1) requires that the waiting element (waiting link) be sufficiently long to remain inactive upon initial loading. However, more space is needed as the waiting element becomes longer and more curved. In this paper, we investigate another configuration, a helicoid, which remedies the spacing issue faced in our previous work. A bistable response under extension can be achieved similar to that of the matrix fracturing in the composite described by Slepyan et al. [2000].

Note on terminology. In this work, we will use the term strip (rather than the earlier "link" or "element") to mean each of the two components of a bistable link: the main strip and the waiting strip. A bistable structure is a chain of bistable links.

Consider a helicoidal bistable link consisting of a main strip and a waiting strip, as in Figure 2. The center core, a solid cylinder, is treated as the main strip, and is made of an isotropic material. The outer layer serves as the waiting strip, and consists of an orthotropic material having its primary direction of orthotropy spiraling about the main strip (central core). By enveloping the inner cylinder in a helicoidal anisotropic shell, the structure maintains symmetry about its cylindrical axis.

Under an axial tensile load, both components of the helicoidal bistable link elongate until the main strip yields. As yielding continues, an increasing portion of the applied load is transferred to the outer helicoidal waiting strip. This paper investigates the energy absorption concept of a bistable structure using a simplified model where the central main strip exhibits linear elastic material behavior to failure (brittle failure). The outer helicoidal waiting strip is assumed to remain nonactive (unloaded) until the
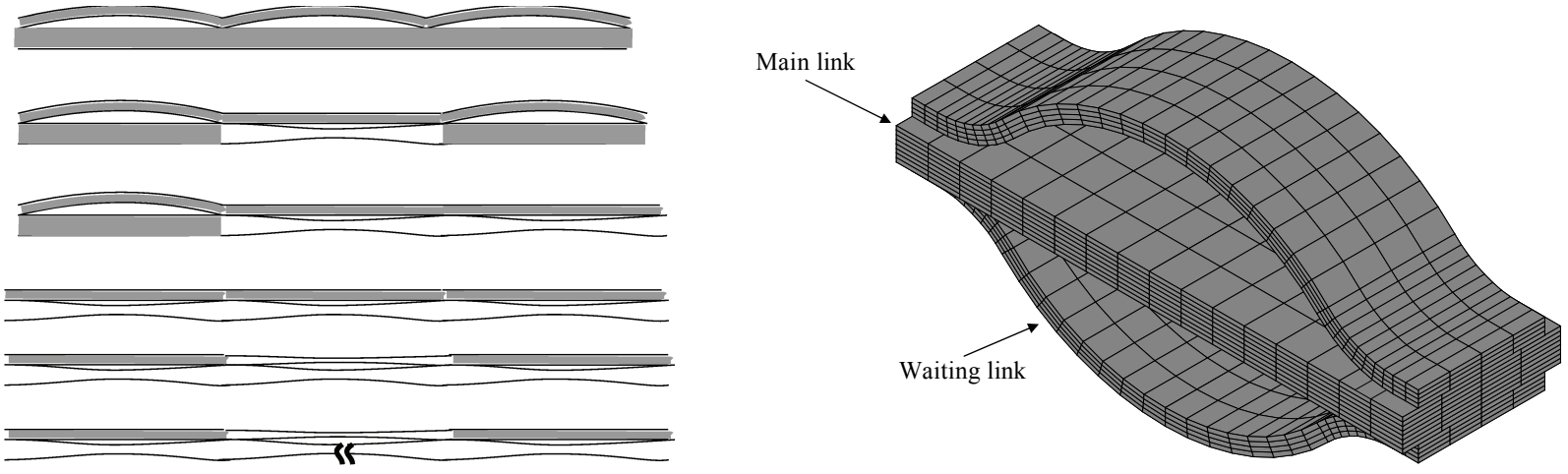

Figure 1. Left: A chain of bistable structures and their damage process. Gray regions represent the undamaged main strips. The broken main strips are shown in white. Right: A bistable structure with a symmetric waiting element. 


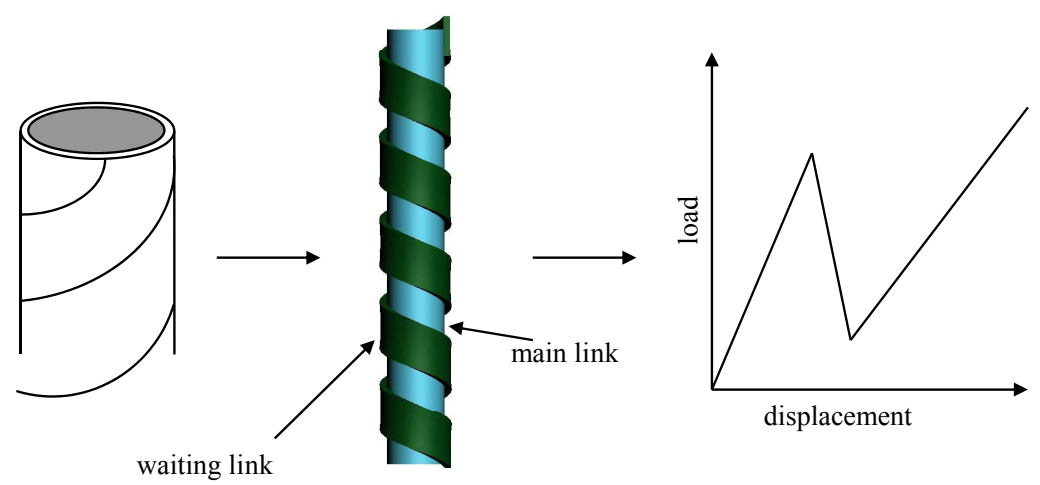

Figure 2. Representative model of a helicoidal bistable structure.

inner main strip fails in a brittle manner. Thus, for a chain of bistable links, each link transitions from a lower strain state to a higher strain state as the main strip (inner cylinder) fails. The main strip produces a transition wave that propagates along the chain. Unlike conventional structures, in which the strain is concentrated near the impact zone, a structure with a waiting strip distributes the strain over a large area.

The outline of this paper is as follows. First, the analytical solutions to the displacements and stresses are computed using the theory of elasticity. The process by which the main strip breaks and the waiting strip becomes active to maintain the structural stability is demonstrated using finite elements. The analytical solutions to the displacements and stresses are used to develop an equivalent one-dimensional discrete model. Three case studies are considered: a single bistable link, a chain of bistable links with sequential breakages of the main strip in each link, and a chain of bistable links with random breakages of the main strip in each link.

\section{Modeling of a helicoidal bistable structure}

To investigate the force-displacement relationship of an individual helicoidal bistable link, a displacement field corresponding to a given applied load is determined from a generalized theory of linear anisotropic elasticity as described in [Lekhnitskii 1981; Leelavanichkul and Cherkaev 2009]. The main strip is made of an isotropic material, while the waiting strip is made of an orthotropic material with its main axis of orthotropy oriented at an angle $\phi$, as shown in Figure 3.

2A. Linear elasticity solution. The structure shown in Figure 3 possesses cylindrical anisotropy; hence, it is more desirable to determine the solution in cylindrical coordinates. The detailed formulation of the solution is given in [Leelavanichkul and Cherkaev 2009]. In three-dimensional stress analysis, stresses and strains are related via Hooke's law in cylindrical coordinates as

$$
\left\{\begin{array}{c}
\sigma_{r r} \\
\sigma_{\theta \theta} \\
\sigma_{z z} \\
\sigma_{\theta z} \\
\sigma_{r z} \\
\sigma_{r \theta}
\end{array}\right\}=\left[\begin{array}{cccccc}
c_{11} & c_{12} & c_{13} & c_{14} & 0 & 0 \\
c_{21} & c_{22} & c_{23} & c_{24} & 0 & 0 \\
c_{31} & c_{32} & c_{33} & c_{34} & 0 & 0 \\
c_{41} & c_{42} & c_{43} & c_{44} & 0 & 0 \\
0 & 0 & 0 & 0 & c_{55} & c_{56} \\
0 & 0 & 0 & 0 & c_{65} & c_{66}
\end{array}\right]\left\{\begin{array}{c}
\epsilon_{r r} \\
\epsilon_{\theta \theta} \\
\epsilon_{z z} \\
\epsilon_{\theta z} \\
\epsilon_{r z} \\
\epsilon_{r \theta}
\end{array}\right\} .
$$



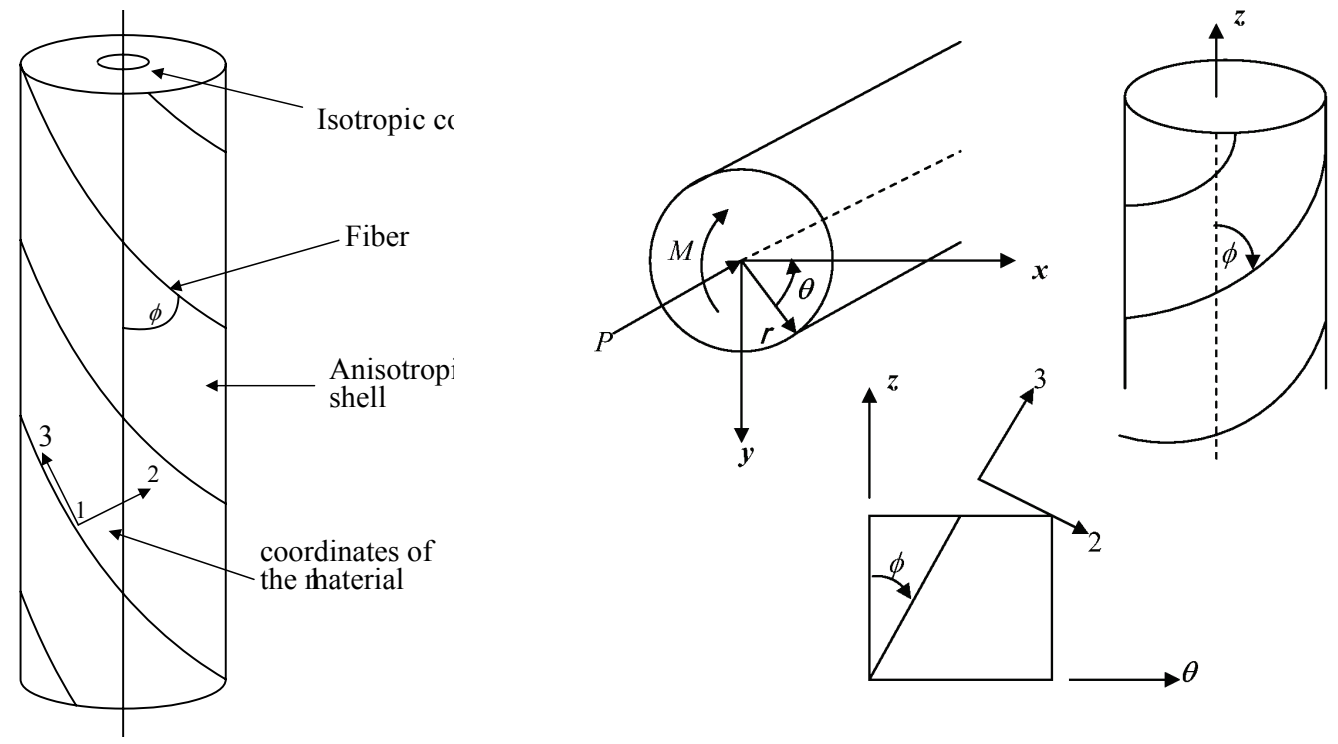

Figure 3. Helicoidal structure and its coordinate system.

Replacing the strains in (2-1) using the strain-displacement relationship

$$
\begin{aligned}
\varepsilon_{r r} & =\frac{\partial u_{r}}{\partial r}, \quad \varepsilon_{\theta \theta}=\frac{1}{r} \frac{\partial u_{\theta}}{\partial \theta}+\frac{u_{r}}{r}, & \varepsilon_{z z} & =\frac{\partial u_{z}}{\partial z}, \\
\varepsilon_{z r} & =\frac{1}{2}\left(\frac{\partial u_{z}}{\partial r}+\frac{\partial u_{r}}{\partial z}\right), & \varepsilon_{\theta z}=\frac{1}{2}\left(\frac{\partial u_{\theta}}{\partial z}+\frac{1}{r} \frac{\partial u_{z}}{\partial \theta}\right), & \left.\frac{1}{r} \frac{\partial u_{r}}{\partial \theta}+\frac{\partial u_{\theta}}{\partial r}-\frac{u_{\theta}}{r}\right),
\end{aligned}
$$

and substituting into the equilibrium equations

$$
\begin{aligned}
& 0=\frac{1}{r} \frac{\partial}{\partial r}\left(r \sigma_{r r}\right)+\frac{1}{r} \frac{\partial}{\partial \theta} \sigma_{r \theta}+\frac{\partial}{\partial z} \sigma_{r z}-\frac{\sigma_{\theta \theta}}{r}, \\
& 0=\frac{1}{r^{2}} \frac{\partial}{\partial r}\left(r^{2} \sigma_{\theta r}\right)+\frac{1}{r} \frac{\partial}{\partial \theta} \sigma_{\theta \theta}+\frac{\partial}{\partial z} \sigma_{\theta z}, \\
& 0=\frac{1}{r} \frac{\partial}{\partial r}\left(r \sigma_{z r}\right)+\frac{1}{r} \frac{\partial}{\partial \theta} \sigma_{z \theta}+\frac{\partial}{\partial z} \sigma_{z z},
\end{aligned}
$$

we obtain the solution to the main strip as

$$
u=C_{1} r, \quad v=\Theta r z, \quad w=C z
$$

and the solution to the waiting strip as

$$
U(r, \theta)=C_{1} r^{k}+C_{2}^{-k}-c_{11} m_{1} r^{2}-4 c_{11} m_{2} r+m_{1} c_{22} r^{2}+m_{2} c_{22} r, \quad V(r, \theta)=\Theta r z, \quad W(r, \theta)=z C,
$$

where

$$
k=\sqrt{\frac{c_{22}}{c_{11}}}, \quad m_{1}=\frac{2 \Theta\left(c_{14}-\frac{1}{2} c_{24}\right)}{\left(c_{22}-c_{11}\right)\left(c_{22}-4 c_{11}\right)}, \quad m_{2}=\frac{C\left(c_{13}-c_{23}\right)}{\left(c_{22}-c_{11}\right)\left(c_{22}-4 c_{11}\right)}
$$


The unknown constants $C, \Theta, C_{1}$, and $C_{2}$ can be found using the boundary conditions

$$
\begin{array}{cccc}
\left.\sigma_{r}\right|_{r_{1}}=\left.\sigma_{r}\right|_{r_{2}}, & \left.\tau_{\theta z}\right|_{r_{1}}=\left.\tau_{\theta z}\right|_{r_{2}}, & \left.\tau_{r z}\right|_{r_{1}}=\left.\tau_{r z}\right|_{r_{2}},\left.\quad \tau_{r \theta}\right|_{r_{1}}=\left.\tau_{r \theta}\right|_{r_{2}}, \\
\left.u(r, \theta)\right|_{r_{1}}=\left.u(r, \theta)\right|_{r_{2}}, & \left.v(r, \theta)\right|_{r_{1}}=\left.v(r, \theta)\right|_{r_{2}}, & \left.w(r, \theta)\right|_{r_{1}}=\left.w(r, \theta)\right|_{r_{2}},
\end{array}
$$

and the end conditions

$$
\begin{gathered}
\int_{0}^{r_{2}} \int_{0}^{2 \pi} \sigma_{z z_{\mathrm{iso}}} r d r+\int_{r_{1}}^{r_{2}} \int_{0}^{2 \pi} \sigma_{z z_{\text {aniso }}} r d r=P, \\
\int_{0}^{r_{2}} \int_{0}^{2 \pi} \sigma_{\theta z_{\mathrm{iso}}} r^{2} d r+\int_{r_{1}}^{r_{2}} \int_{0}^{2 \pi} \sigma_{\theta z_{\text {aniso }}} r^{2} d r=0,
\end{gathered}
$$

where $r_{1}$ is the radius of the main strip and $r_{2}$ is the outer radius of the waiting strip. Once all the unknown constants are determined, Equations (2-4) and (2-5) can be substituted into (2-2) to obtain the strains, after which the stresses can be computed using (2-1).

2B. Simulation of breakages using the finite element method. While analytical solutions to the displacements and stresses of the undamaged link are obtained from the theory of anisotropic elasticity, we investigate the breakages of the main strip using the finite element method. Our analytical solution gives stresses based on the assumption that the strains do not vary along the structure's generator. Hence, the solution obtained cannot describe how stresses are distributed along the longitudinal direction of the structure when the length of the structure is finite or when there is a boundary effect from a discontinuity. A single breakage in a bistable link is simulated to show the effect that the spiraling angle $\phi$ (see Figure 3) has on the force-displacement relationship. Then, a simulation where multiple breakages occur is performed to determine the minimum length of each link such that stress interactions due to breakages of the main strips are minimal. Notice that for simplicity we do not consider stress interactions in neighboring breakage points, and we assume that the failure occurs only in the main strips.

To simulate the failure of the main strip, two load steps are used. The first load step is applied to the point where failure of the main strip occurs. The second load step begins following the localized failure of the main strip. Each of these two load steps consists of several substeps that discretely update the stiffness matrix.

Example 2.1: Numerical simulation of a breakage in the main strip. A three-dimensional finite element model of the structure is used and its undamaged model is verified with an analytical solution as shown in Figure 4. For demonstration purposes, we consider a bistable structure made from materials with the following properties:

- The main strip's isotropic material has a shear modulus of $25.51 \mathrm{GPa}$ and a Poisson's ratio of 0.35 .

- The waiting strip is modeled using an anisotropic material having a helicoidal fiber orientation and the following material properties:

\begin{tabular}{|lcccccc|}
\hline Parameter & $E_{11}=E_{22}$ & $E_{33}$ & $G_{23}=G_{13}$ & $G_{12}$ & $v_{23}=v_{13}$ & $v_{12}$ \\
Value & $7.591 \mathrm{GPa}$ & $139.348 \mathrm{GPa}$ & $3.789 \mathrm{GPa}$ & $2.782 \mathrm{GPa}$ & 0.0178 & 0.364 \\
\hline
\end{tabular}



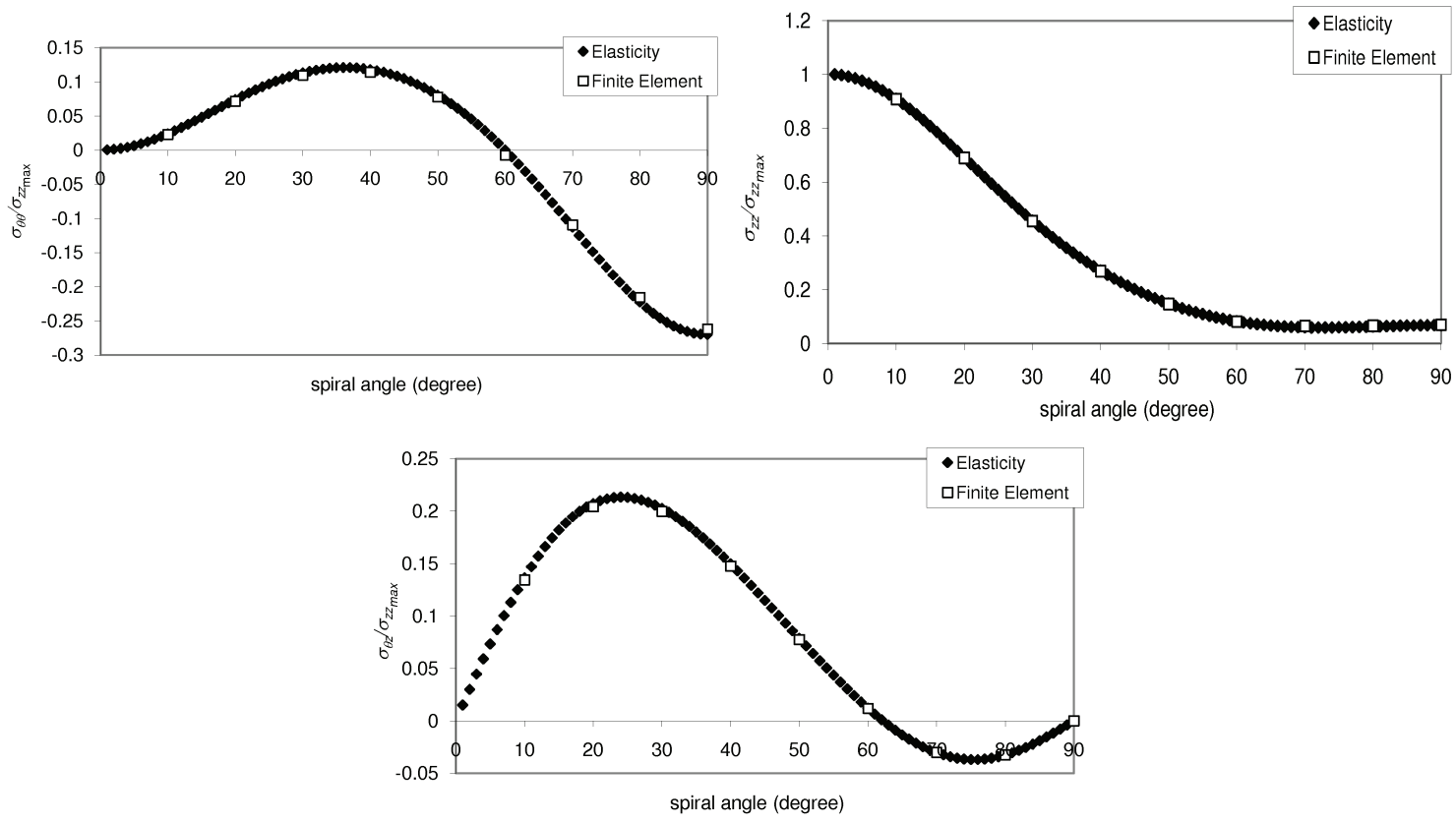

Figure 4. Verification of the finite element model.

- The radius of the inner cylinder is $6.35 \mathrm{~mm}$ and the thickness of the outer shell is $1.59 \mathrm{~mm}$. Only axial tensile loading is considered.

The force versus displacement response for a range of fiber angles in the helicoidal waiting strip is shown in Figure 5. The effect of the main strip breakage (produced at an applied displacement of $0.127 \mathrm{~mm}$ ) increases as the fiber angle increases. As the fiber angle increases, the axial stiffness of the waiting strip decreases as evidenced by the decreasing slope in Figure 5. This behavior is desirable in order to achieve the desired bistable response.

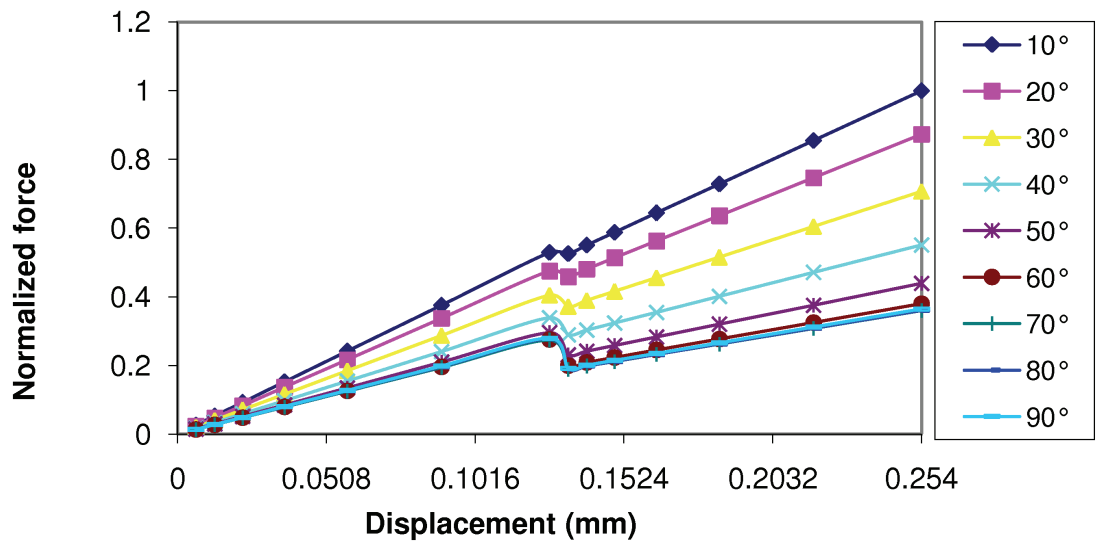

Figure 5. Force-displacement of the bistable model with a single damage. The force is normalized by the maximum force when the displacement is $0.254 \mathrm{~mm}$. 
Ideally, the waiting strip should have no effect on the structural response until the main strip fails. At lower fiber angles, the waiting strip becomes stiffer and requires a higher applied load to achieve the same axial displacement. The dips in the curves shown in Figure 5 represent the amount of energy dissipated as a result of the main strip breakage. Hence, less energy is dissipated when the spiraling angle is small.

Example 2.2: Stress distribution around the broken area of the main strip. The stress field near the broken area in the main strip is investigated in this example to determine the stress distribution when three-dimensional effects are considered. When more than one damage point is expected, the distance between each damage point must be determined such that the stresses in the neighborhoods of these points do not interact with each other. The objective of this part is to determine the most basic condition which simplifies the analysis of the breakage; namely, to avoid any complication of the stress riser due to the finite region. We investigate the distribution of the stresses near the broken area in the main strip. Using the model discussed in the previous section, the von Mises stresses of each variation of the angle of orthotropy and applied loads are computed for the main strip material and shown in Figure 6.

The stresses shown in these figures are normalized to their corresponding maximum stress values. Roughly, three groups of stress results can be seen in these figures. They represent when the fiber angles are $10^{\circ}, 30^{\circ}$, and $\geq 50^{\circ}$. Each line within each group represents the result for a different magnitude of the applied load. These figures illustrate that the interval of the high stress concentration $x_{0}$ does not depend on the magnitude of the applied load nor the spiraling angle. For the structural configuration in Example 2.1, $x_{0}$ is approximately $12.7 \mathrm{~mm}$.

When there are multiple locations of the damage, as shown in Figures 7 and 8, the interactions of the concentrated stresses are still minimal as long as the broken locations take place $25.4 \mathrm{~mm}$ from each other. Here, breakages are introduced to seven locations simultaneously, $50.8 \mathrm{~mm}$ apart from each other. One can see that the distribution of stresses around the breakages has the same characteristics, and they do not interact with one another.

Next, the effect due to the change of the radius $r_{i}$ of the main strip is investigated, with the total cross-sectional area of the structure held constant.

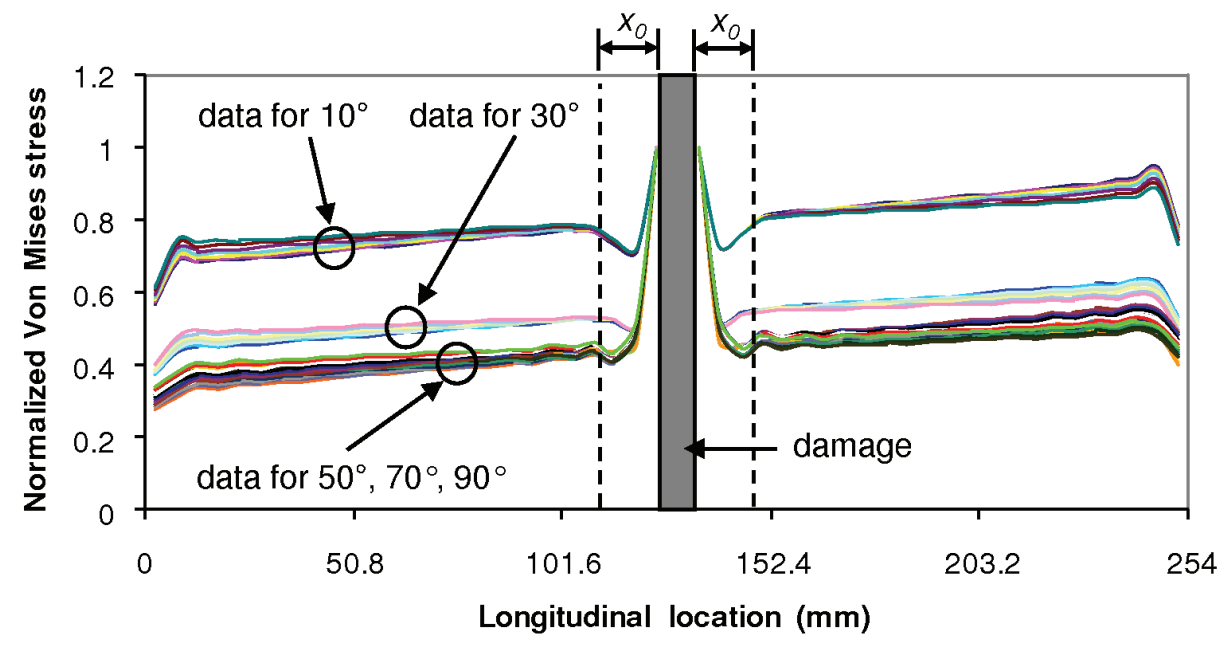

Figure 6. Normalized von Mises stress around the breakage. 


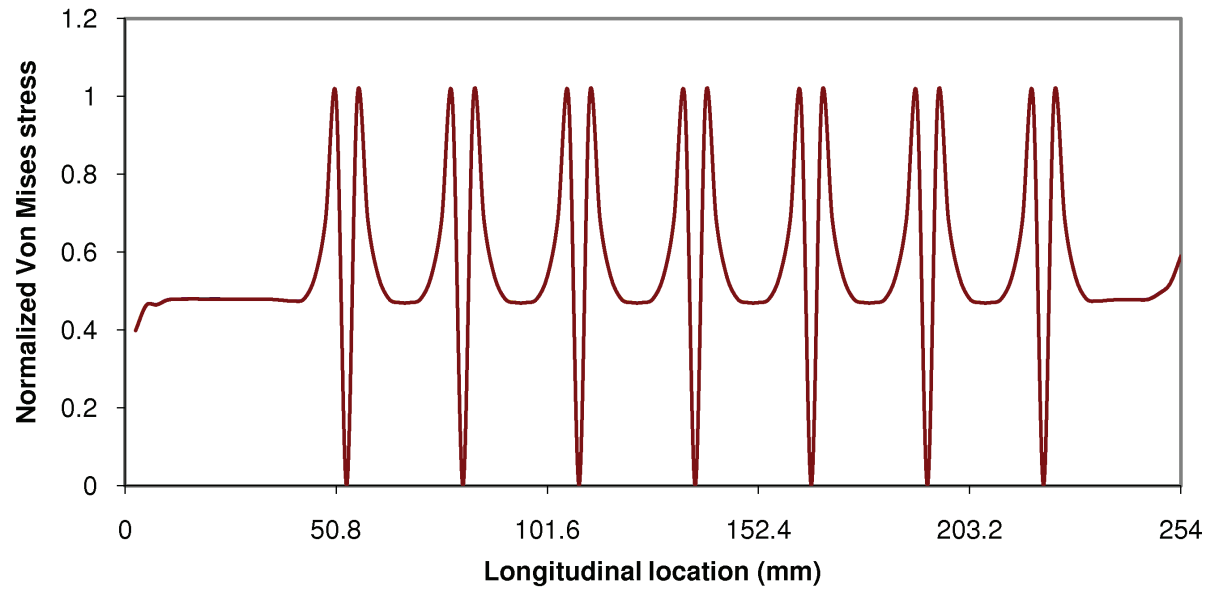

Figure 7. Stress distribution when the breakage locations are $50.8 \mathrm{~mm}$ apart $\left(60^{\circ}\right)$.

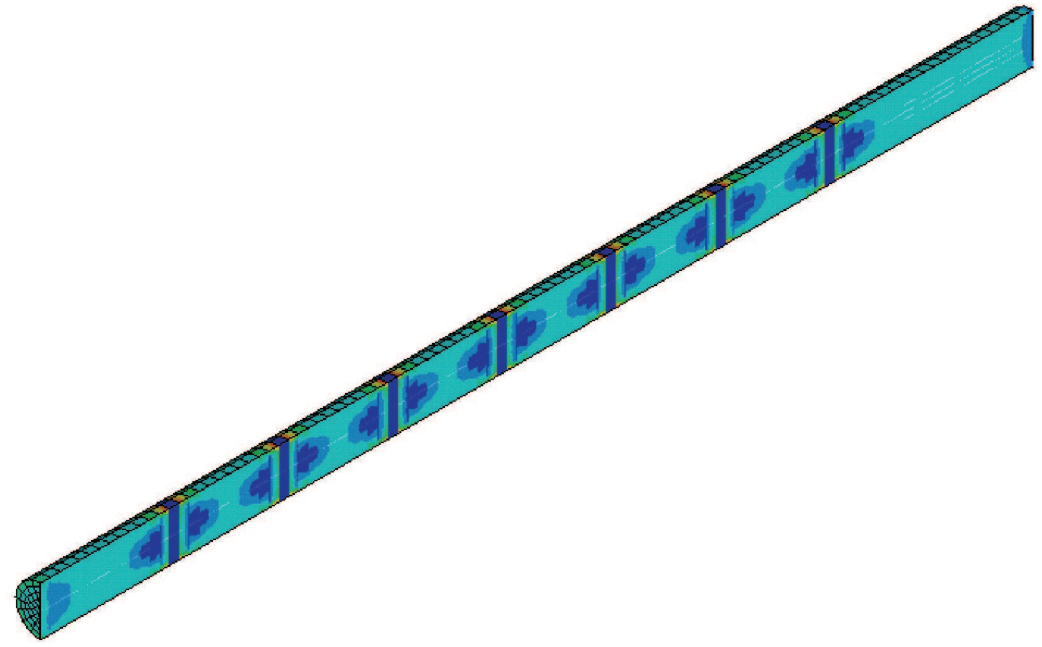

Figure 8. Contour plot of normalized von Mises stress in the main link when the breakage locations are $50.8 \mathrm{~mm}$ apart. Darker (blue) areas correspond to values near 0 and lighter areas to values near 0.3 .

Example 2.3: Effect of changes in the radii of the main strip and waiting strip. The normalized von Mises stress in the center core is shown in Figure 9, top. The legends there represent the ratio between the radii of the waiting strip and the main strip, $r_{o} / r_{i}$. The pattern of stress distributions cannot be obtained from these plots due to the fluctuation of the stresses. The fluctuation of the von Mises stress becomes more visible at lower $r_{o} / r_{i}$ ratios. These oscillations are the result of the approximation of the element stress. However, the differences in the magnitudes between peaks are reduced, but remain, as the number of elements is increased while the results converge. Each period of the oscillation corresponds to the length of the element, which $5.08 \mathrm{~mm}$.

To further investigate the distribution of the stresses, two more scenarios are investigated. The first occurs when the main strip's radius remains fixed $6.35 \mathrm{~mm}$ and the radius of the waiting strip is varied. 

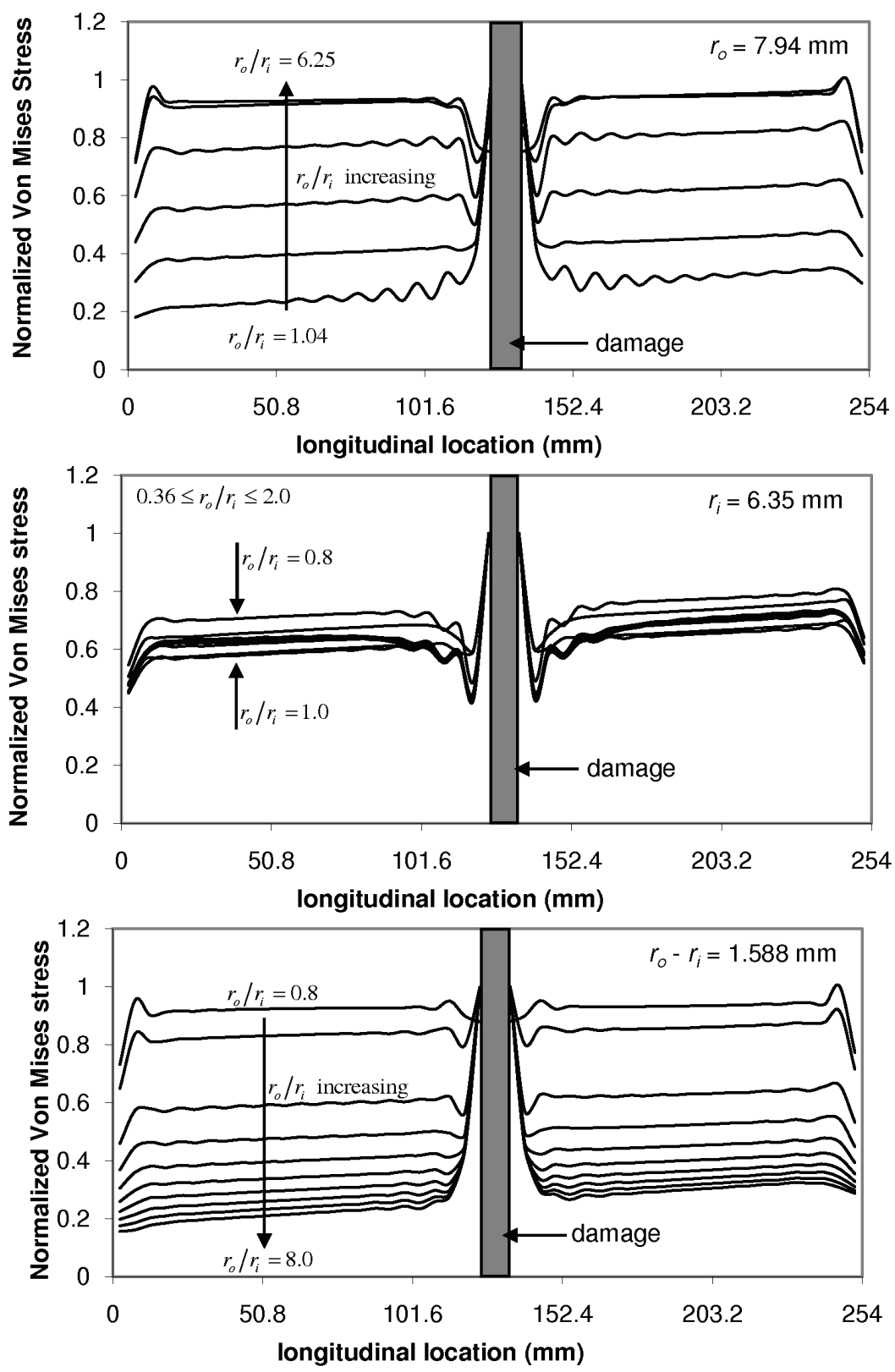

Figure 9. Stress distribution around the breakage in the main link. Top: $r_{i}$ is varied while $r_{o}$ remains at $7.94 \mathrm{~mm}$. Middle: $r_{o}$ is varied while $r_{i}$ remains at $6.35 \mathrm{~mm}$. Bottom: $r_{i}$ and $r_{o}$ are varied while the thickness of the waiting link is held at $1.59 \mathrm{~mm}$.

The second case occurs when the thickness of the waiting strip is fixed at $1.59 \mathrm{~mm}$ and the radius of the main strip is varied. Results for these two cases are shown in the last two parts of Figure 9.

These results show that the stress distribution depends greatly on the ratio of the inner and outer radii of the spiraling layer, $r_{o} / r_{i}$. For the specific model having the properties as given in Example 2.1, the 


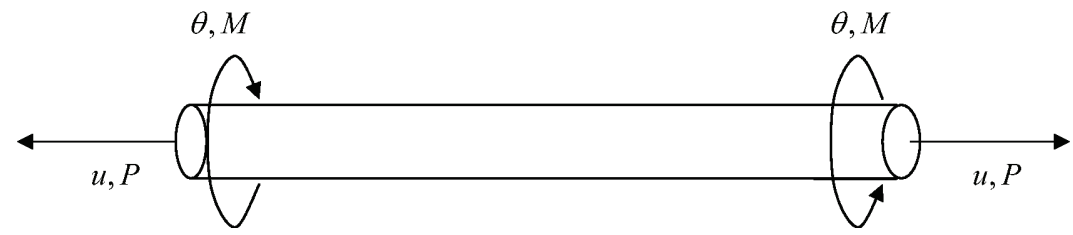

Figure 10. One-dimensional structural model.

range of $x_{0}$ values from three-dimensional analysis is $25.4 \mathrm{~mm}$. This value, however, may change as the geometry of the structure varies. To maintain our focus on the bistable structure and its energy absorption capabilities, we did not analyze further the three-dimensional stress effect.

\section{One-dimensional models}

Since the deformations of the structure considered in this paper are symmetric about a cylindrical axis, this structure can be represented by a one-dimensional model. It is convenient to replace the threedimensional cylinder with a link. The helicoidal symmetry results in a coupling between a rotation about the axis and the elongation as shown in Figure 10.

3A. One-dimensional structural model. A one-dimensional structural element is developed based on the formulation given in [Leelavanichkul and Cherkaev 2009], where an elongation is coupled to a rotation. Linkages are useful in describing the physics of a larger structure assembled from multiple links where the displacement and reactions at their ends are of major interest. We obtain the stiffness matrix $\boldsymbol{k}$ relating the force $P$ and moment $M$ at the element ends to the elongation and the rotation about the $\theta$-axis:

$$
\boldsymbol{f}=\boldsymbol{k} \boldsymbol{u} \quad \text { or } \quad\left\{\begin{array}{c}
P \\
M
\end{array}\right\}=\left[\begin{array}{ll}
k_{1} & k_{2} \\
k_{4} & k_{3}
\end{array}\right]\left\{\begin{array}{l}
u \\
\theta
\end{array}\right\} .
$$

The quantities of interest are the stiffness constants $k_{1}, k_{2}, k_{3}$, and $k_{4}$. Without losing generality, one can assume that $k_{4}=k_{2}$. Using the displacements, which are given by (2-4) and (2-5), at two measures of the loads $P$ and $M$, the stiffness $k$ constants can be determined [Leelavanichkul and Cherkaev 2009].

Example 3.1: Illustration of the varying stiffnesses as function of angle $\phi$. The stiffness values of the equivalent one-dimensional model of the structure given in Example 2.1 are illustrated in Figure 11. These plots demonstrate how the stiffness values vary with the spiral angle. The values of the stiffness also vary linearly with the load $P$ and the length $L$ of the three-dimensional model. Despite having only an axial load applied, the stiffness constants $k_{2}$ and $k_{3}$ generate rotation as the structure is stretched. We realize that the effect of the coupling increases the energy release capability while the elongation remains the same. The additional twisting degrees of freedom correspond to additional energy released in the process of elongation.

3B. Finite element model: formulation of the stiffness matrix. A single element, as shown in Figure 12, used in the finite element model can be constructed based on the one-dimensional structural model given in the previous section including both elongation and rotational degrees of freedom. When the structure is subjected to an axial load, the force-displacement relation is governed by

$$
\boldsymbol{F}=\boldsymbol{K}(\boldsymbol{u}) \boldsymbol{u}
$$




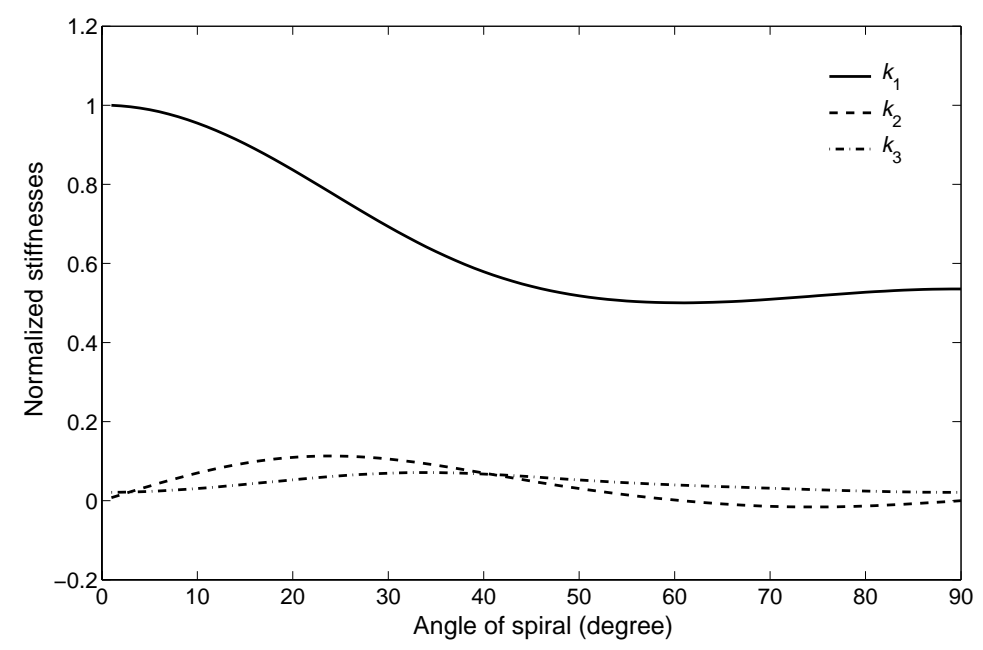

Figure 11. Stiffnesses of the one-dimensional model (normalized by the maximum value of $k_{1}$ ).

where

$$
\boldsymbol{K}(\boldsymbol{u})= \begin{cases}\boldsymbol{K}_{1} & \text { if } \sigma_{e} \leq \sigma_{Y}, \\ \boldsymbol{K}_{2} & \text { otherwise, }\end{cases}
$$

and $\sigma_{e}$ and $\sigma_{Y}$ represent the stress in the element and the main strip yield stress, respectively. The stiffness matrix $\boldsymbol{K}_{1}$ is the total stiffness of the bistable structure and the stiffness matrix $\boldsymbol{K}_{2}$ is the stiffness of the waiting strip only. The axial deformation is coupled with the rotation about the generator; see Figure 12.

Thus, the deformations are represented by

$$
\delta=\hat{d}_{2 x}-\hat{d}_{1 x}, \quad \gamma=\hat{\phi}_{2 x}-\hat{\phi}_{1 x},
$$

where $\delta$ and $\gamma$ are the elongation and rotation, respectively. The local axial displacement is represented by $\hat{d}$ while the nodal rotation is represented by $\hat{\phi}$. The force-displacement relationship is expressed as

$$
P=k_{1} \delta+k_{2} \gamma, \quad M=k_{2} \delta+k_{3} \gamma,
$$

where $P$ and $M$ are the axial and torsional loads, respectively. The quantities $k_{1}, k_{2}$, and $k_{3}$ are the stiffness constants. Substituting (3-2) into (3-3), we have

$$
P=k_{1}\left(\hat{d}_{2 x}-\hat{d}_{1 x}\right)+k_{2}\left(\hat{\phi}_{2 x}-\hat{\phi}_{1 x}\right), \quad M=k_{2}\left(\hat{d}_{2 x}-\hat{d}_{1 x}\right)+k_{3}\left(\hat{\phi}_{2 x}-\hat{\phi}_{1 x}\right) .
$$

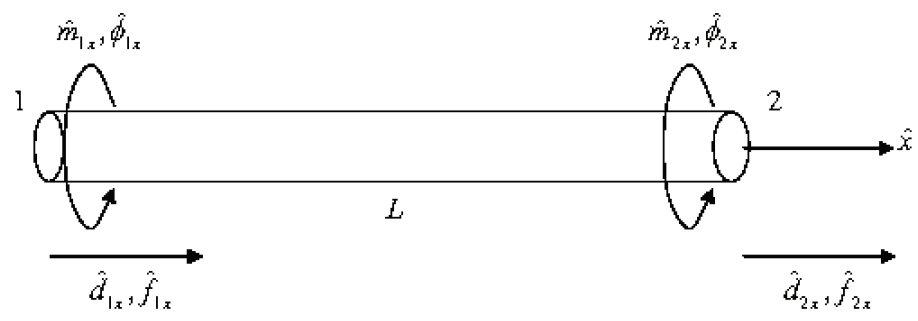

Figure 12. One-dimensional structural element. 
By the sign convention for the nodal forces and equilibrium,

$$
\hat{f}_{1 x}=-P, \quad \hat{f}_{2 x}=P, \quad \hat{m}_{1 x}=M, \quad \hat{m}_{2 x}=-M .
$$

Substituting (3-4) into (3-5), the following system is constructed:

$$
\left\{\begin{array}{c}
\hat{f}_{1 x} \\
\hat{m}_{1 x} \\
\hat{f}_{2 x} \\
\hat{m}_{2 x}
\end{array}\right\}=\left[\begin{array}{rrrr}
k_{1} & k_{2} & -k_{1} & -k_{2} \\
k_{2} & k_{3} & -k_{2} & -k_{3} \\
-k_{1} & -k_{2} & k_{1} & k_{2} \\
-k_{2} & -k_{3} & k_{2} & k_{3}
\end{array}\right]\left\{\begin{array}{l}
\hat{d}_{1 x} \\
\hat{\phi}_{1 x} \\
\hat{d}_{2 x} \\
\hat{\phi}_{2 x}
\end{array}\right\} .
$$

Hence, the element stiffness matrix is defined as

$$
\hat{\boldsymbol{K}}=\left[\begin{array}{rrrr}
k_{1} & k_{2} & -k_{1} & -k_{2} \\
k_{2} & k_{3} & -k_{2} & -k_{3} \\
-k_{1} & -k_{2} & k_{1} & k_{2} \\
-k_{2} & -k_{3} & k_{2} & k_{3}
\end{array}\right] \text {, }
$$

where $k_{1}, k_{2}$, and $k_{3}$ are the values calculated from the one-dimensional structural model as discussed in Section 3 .

Two breakage scenarios are investigated: a sequential breakage and a random breakage. Both scenarios are demonstrated in this paper using numerical examples based on the formulation discussed above. Considering the structure given in Example 2.1, breakages of the main strip are assumed to occur at $50.8 \mathrm{~mm}$ intervals for this particular example to avoid interaction of concentrated stresses [Leelavanichkul 2007].

Example 3.2: Sequential breakages. For the case of sequential breakage of the main strip (central core), each new breakage occurs adjacent to the previous one. The location of the new core breakage is assumed to be $50.8 \mathrm{~mm}$ from the preceding core breakage as shown in Figure $13\left(2 x_{0}=50.8\right)$. The objective of this analysis is to determine the total energy absorption during the sequence of core breakages.

The material of the main strip is assumed to return to its unloaded state after a breakage occurs. The stiffness $\boldsymbol{K}(u)$ of the damaged link is updated with the new values $\boldsymbol{K}_{2}$ obtained from the computation of the one-dimensional structural model. We treat the main strip as an elastic-brittle material, therefore, the von Mises failure criterion is chosen to determine when the failure of the main strip occurred. Axial

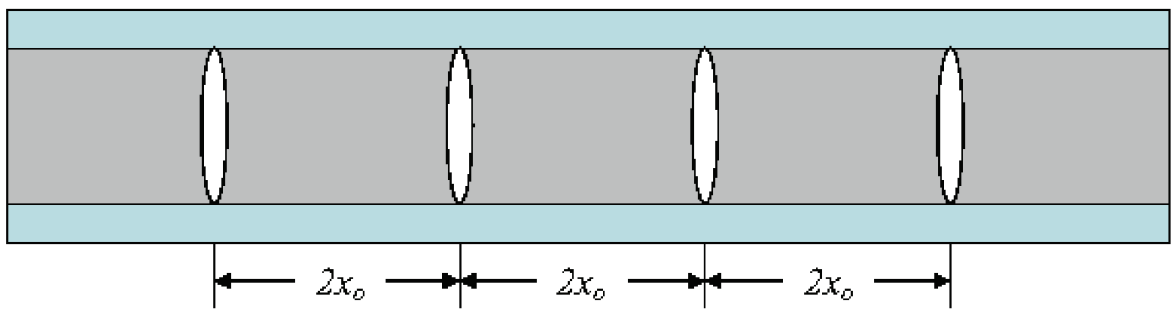

Figure 13. Representative model of sequential breakages. 


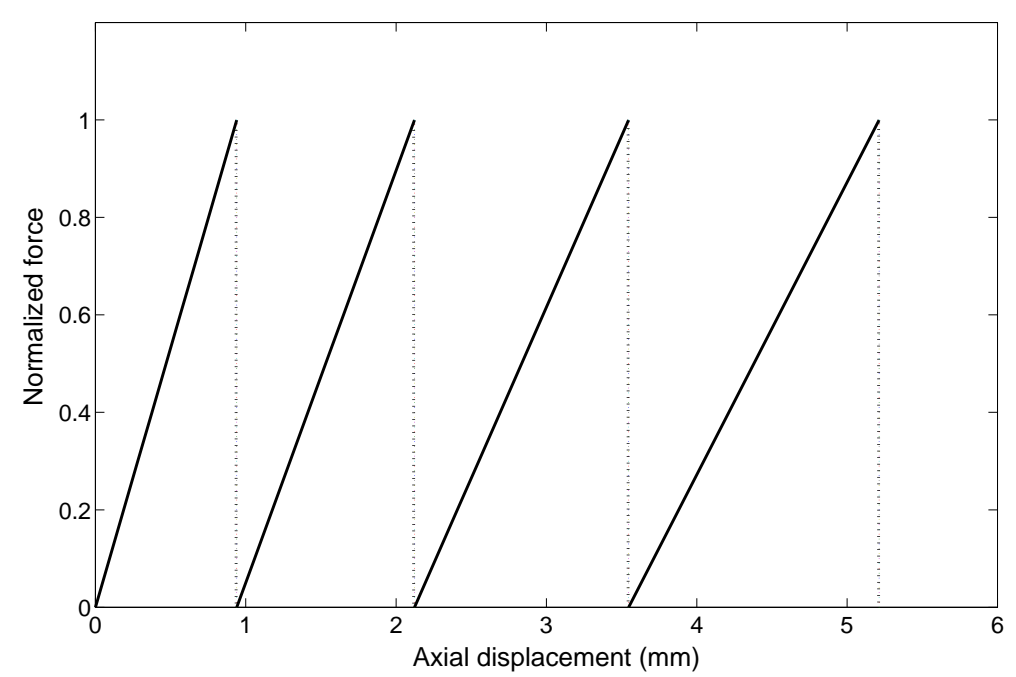

Figure 14. Force-displacement diagram for three sequential core breakages of a $254 \mathrm{~mm}$ axially loaded chain consisting of twenty links.

and shear stresses are determined separately using the nodal displacements,

$$
\sigma_{x}=E \varepsilon_{x}=E \frac{d u}{d x}=\frac{E}{L}\left(\hat{d}_{2 x}-\hat{d}_{2 x}\right), \quad \tau_{x y}=G \gamma_{x y}=\frac{G r}{L}\left(\hat{\phi}_{2 x}-\hat{\phi}_{2 x}\right) .
$$

The applied load is increased until the material yield strength is met:

$$
\sigma_{e}=\sqrt{\sigma_{1}^{2}-\sigma_{1} \sigma_{2}+\sigma_{2}^{2}} \leq \sigma_{Y},
$$

where $\sigma_{e}$ is the element von Mises stress and $\sigma_{Y}$ is the material yield stress. The principal stresses $\sigma_{1}$ and $\sigma_{2}$ are expressed as

$$
\sigma_{1,2}=\frac{\sigma_{x}}{2} \pm \sqrt{\left(\frac{\sigma_{x}}{2}\right)^{2}+\tau_{x y}^{2}} .
$$

The force versus displacement response for the sequential breakage of a chain composed of four helicoidal bistable structures, each with a waiting strip having a fiber angle of $60^{\circ}$, is illustrated in Figure 14 . Three main strip breakages take place in the chain (represented by vertical lines in the figure). Each time a breakage occurs, the overall stiffness of the structure is reduced and an increase in displacement is required for the next breakage to occur. The total strain energy of $31 \mathrm{~J}$ is required to produce three breakages for the chosen materials and specimen geometry.

Due to the anisotropy of the waiting strips, the conventional maximum strength criterion for isotropic materials gives a poor prediction of the failure. We use the Tsai-Hill failure criterion to determine whether the outer helicoidal layer fails:

$$
\left(\frac{\sigma_{1}}{\sigma_{1 u}}\right)^{2}+\left(\frac{\sigma_{2}}{\sigma_{2 u}}\right)^{2}-\frac{\sigma_{1} \sigma_{2}}{\sigma_{1 u}^{2}}+\left(\frac{\tau_{12}}{\tau_{12 u}}\right)^{2}<1 .
$$

When the left side is reaches or exceeds 1 , failure is predicted. For an angle of $60^{\circ}$, the predicted values are approximately 0.2 and 0.45 at the outer and inner surfaces, respectively, of the helicoidal layer. 


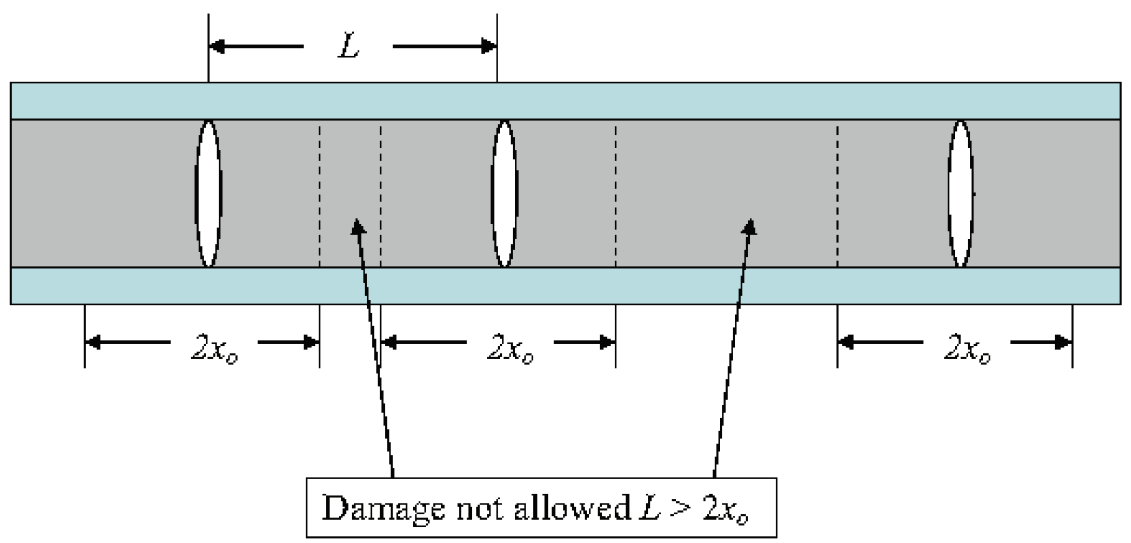

Figure 15. Representative model of random breakages.

The Tsai-Hill failure criterion does not distinguish between tensile and compressive failures, but is sufficient for our goal. The waiting strip becomes active only after the main strip fails. The nature of the main strip failure is not considered here. Axial and shear stresses computed in the element are used in the Tsai-Hill failure criterion.

Example 3.3: Random breakages. The breakages of the main strip may not necessarily occur sequentially. For nonsequential breakages, the same requirement for the spacing $(50.8 \mathrm{~mm})$ between the main strip breakages as in the sequential analysis is imposed. As shown in Figure 15, the spacing $x_{0}$ can become as large as $101.6 \mathrm{~mm}$ for the same structure used in the previous section.

Consider a vector $\boldsymbol{A}$ containing elements 1 to $n,\left[e_{1}, e_{2}, e_{3}, \ldots, e_{n}\right]$, as shown in Figure 16 . The random breakages are assumed to be uniformly distributed over the interval $[1, n]$. Each time an element is selected, the load is incrementally applied and the von Mises stress is computed. Once the yield strength of the selected element is reached, that element is presumed to have failed. The elements within the range $25.4 \mathrm{~mm}$ to the left and to the right of the broken element are then removed from the vector $\boldsymbol{A}$. The next randomly selected element is taken from the new updated $\boldsymbol{A}$. The process is repeated until no elements are left in the updated vector $\boldsymbol{A}$.

Using the stiffness matrix formulated in Section 3, the resulting force versus displacement response is presented in Figure 17 for the case when three breakages occur before the chain of consisting of four bistable structures completely fails. The main strip (inner core) breakages occur at the following positions from the fixed end: $145 \mathrm{~mm}, 66 \mathrm{~mm}$, and finally $201 \mathrm{~mm}$. Despite the random sequence of main strip breakages, the same force-displacement diagram is produced as for the case of sequential breakages.

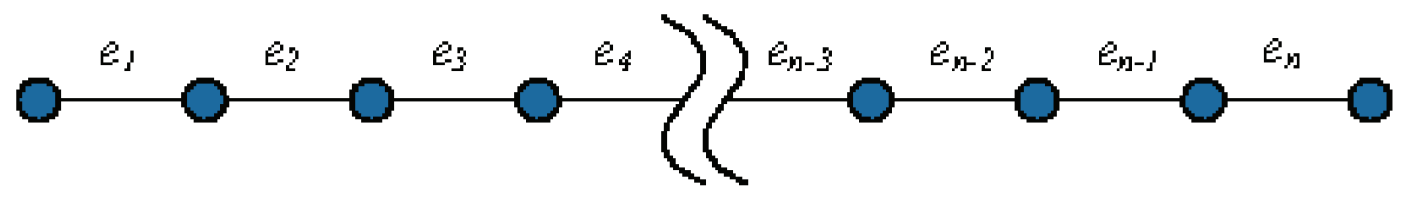

$$
\mathbf{A}=\left[e_{1}, e_{2}, e_{3}, e_{4}, \ldots, e_{n-3}, e_{\mathrm{m}-2}, e_{n-1}, e_{\mathrm{n}}\right]
$$

Figure 16. Elements as components of vector $\boldsymbol{A}$. 

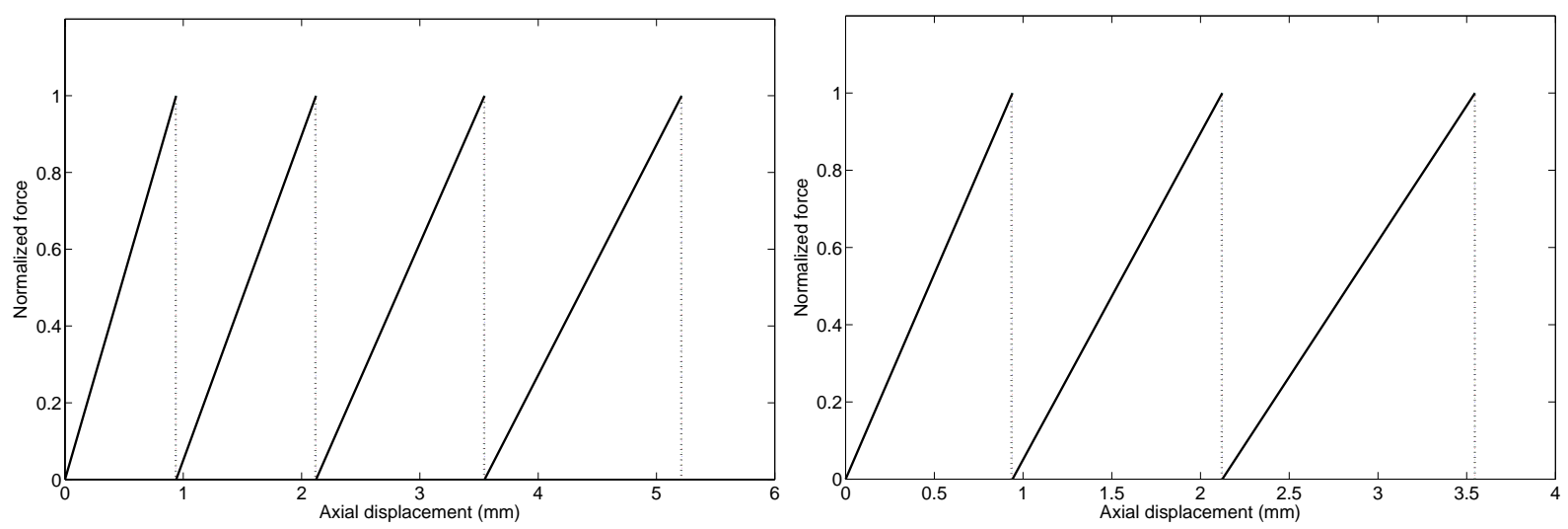

Figure 17. Force-displacement diagram for random breakages: three breakages (left) and two breakages (right).

The total strain energy required to produce three random breakages, $31.0 \mathrm{~J}$, is also the same as for the case of sequential breakages.

The results presented in Figures 14 and 17 show that the order in which the core breakages occur does not affect the force versus displacement response of the structure. Thus, the amount of energy absorbed depends only on the number of the breakages - since the same amount of strain energy per unit volume is required to break the helicoidal main strip.

A comparison of the energy absorbed by the structure in this investigation to a conventional structure of the same size and geometry made of only isotropic material is presented here:

\begin{tabular}{|ll|}
\hline Conventional structure & $7.74 \mathrm{~J}$ \\
Bistable structure (sequential) & $31.0 \mathrm{~J}$ \\
Bistable structure (random) & $24.0-31.0 \mathrm{~J}$ \\
\hline
\end{tabular}

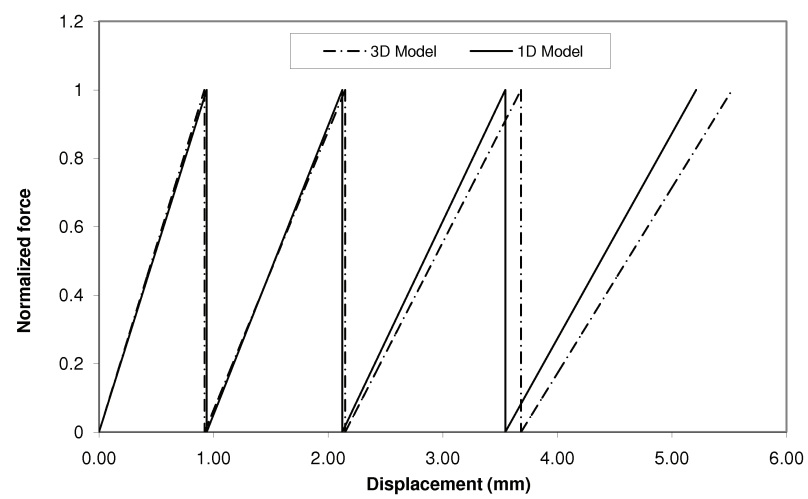

Figure 18. Comparison of force-displacement relation between one- and threedimensional models. The results show good agreement for small deformations. 
A comparison of the force-displacement relation between the two models is shown in Figure 18. This comparison illustrates that the simplified one-dimensional model gives an approximation of the force-displacement relation close to that of the three-dimensional model only when the deformation is sufficiently small. The advantage of the one-dimensional finite element model is the relatively low number of elements required in comparison to the three-dimensional model. Hence, the computational time is reduced significantly, which is extremely beneficial to the design, optimization, and concept development process.

\section{Conclusion}

A helicoidal bistable structure is proposed and its energy absorption behavior studied. In comparison with the earlier bistable structure studied in [Cherkaev et al. 2006], the helicoidal bistable structure allows for a longer waiting inactive time relative to the additional spacing required, It can also be designed to sustain a smaller elongation for the same energy release. The additional twisting degrees of freedom correspond to the additional energy released in the process of elongation.

A one-dimensional model is formulated for numerical simulations of a linear chain of helicoidal bistable links. The location where the breakages occur does not affect the amount of the energy absorbed. Comparison of the force-displacement relations shows that the one-dimensional model gives a good approximation of the three-dimensional model when the deformation is sufficiently small. The helicoidal bistable structure can absorb more energy, in comparison to the conventional structure, by distributing the damage along its length.

\section{Acknowledgements}

Support of this work by the Army Research Office (ARO) is gratefully acknowledged. Helpful insights from the reviewers and the editor have significantly improved the exposition of the paper.

\section{References}

[Balk et al. 2001a] A. M. Balk, A. V. Cherkaev, and L. I. Slepyan, "Dynamics of chains with non-monotone stress-strain relations, I: model and numerical experiments", J. Mech. Phys. Solids 49:1 (2001), 131-148.

[Balk et al. 2001b] A. M. Balk, A. V. Cherkaev, and L. I. Slepyan, "Dynamics of chains with non-monotone stress-strain relations, II: nonlinear waves and waves of phase transition", J. Mech. Phys. Solids 49:1 (2001), 149-171.

[Cherkaev and Slepyan 1995] A. Cherkaev and L. Slepyan, "Waiting element structures and stability under extension", Int. J. Damage Mech. 4:1 (1995), 58-82.

[Cherkaev and Zhornitskaya 2003] A. Cherkaev and L. Zhornitskaya, "Dynamics of damage in two-dimensional structures with waiting links", pp. 273-284 in IUTAM symposium on Asymptotics, Singularities and Homogenisation in Problems of Mechanics (Liverpool, United Kingdom, 2002), edited by A. B. Movchan, Solid mechanics and its applications, Kluwer, Dordrecht, 2003.

[Cherkaev et al. 2005] A. Cherkaev, E. Cherkaev, and L. Slepyan, "Transition waves in bistable structures, I: delocalization of damage", J. Mech. Phys. Solids 53:2 (2005), 383-405.

[Cherkaev et al. 2006] A. Cherkaev, V. Vinogradov, and S. Leelavanichkul, "The waves of damage in elastic-plastic lattices with waiting links: design and simulation", Mech. Mater. 38:8-10 (2006), 748-756.

[Leelavanichkul 2007] S. Leelavanichkul, Helicoidal morphology in engineering structures, Ph.D. thesis, University of Utah, Salt Lake City, 2007. 
[Leelavanichkul and Cherkaev 2009] S. Leelavanichkul and A. Cherkaev, "Elastic cylinder with helicoidal orthotropy: theory and applications", Int. J. Eng. Sci. 47:1 (2009), 1-20.

[Lekhnitskii 1981] S. G. Lekhnitskii, Theory of elasticity of an anisotropic body, Mir Publishers, Moscow, 1981.

[Slepyan and Tronyankina 1988] L. I. Slepyan and L. V. Tronyankina, "Impact waves in a nonlinear chain", Strength and Visco-plasticity (1988), 301-305. in Russian.

[Slepyan and Troyankina 1984] L. I. Slepyan and L. V. Troyankina, "Fracture wave in a chain structure", J. Appl. Mech. Tech. Phys. 25:6 (1984), 921-927.

[Slepyan et al. 2000] L. I. Slepyan, V. Krylov, and R. Parnes, "Helical inclusion in an elastic matrix", J. Mech. Phys. Solids 48:4 (2000), 827 - 865 .

[Slepyan et al. 2005] L. Slepyan, A. Cherkaev, and E. Cherkaev, "Transition waves in bistable structures, II: analytical solution: Wave speed and energy dissipation”, J. Mech. Phys. Solids 53:2 (2005), 407-436.

[Whitman and La Saponara 2007a] Z. Whitman and V. La Saponara, "Bistable structures for energy absorption, I: metallic structures under tension", J. Mech. Mater. Struct. 2:2 (2007), 347-358.

[Whitman and La Saponara 2007b] Z. Whitman and V. La Saponara, "Bistable structures for energy absorption, II: composite structures under tension”, J. Mech. Mater. Struct. 2:2 (2007), 359-376.

Received 6 Feb 2009. Revised 23 Jun 2009. Accepted 20 Aug 2009.

SEUBPONG LEELAVANiCHKUL: sleelava@eng.utah.edu

Department of Mechanical Engineering, University of Utah, Salt Lake City, UT 84112, United States

ANDREJ CHERKAEV: cherk@math.utah.edu

Department of Mathematics, University of Utah, 155 S. 1400 E., Room 233, Salt Lake City, UT 84112, United States

DANIEL O. ADAMS: adams@mech.utah.edu

Department of Mechanical Engineering, University of Utah, Salt Lake City, UT 84112, United States

FLORIAN SOLZBACHER: solzbach@ece.utah.edu

Department of Electrical \& Computer Engineering, University of Utah, Salt Lake City, UT 84112, United States 


\title{
JOURNAL OF MECHANICS OF MATERIALS AND STRUCTURES
}

\author{
http://www.jomms.org
}

\author{
Founded by Charles R. Steele and Marie-Louise Steele

\section{EDITORS} \\ Charles R. STEele \\ DAVIDE BIGONI \\ IWONA JASIUK \\ YASUHIDE SHINDO \\ Stanford University, U.S.A. \\ University of Trento, Italy \\ University of Illinois at Urbana-Champaign, U.S.A. \\ Tohoku University, Japan
}

\section{EDITORIAL BOARD}

H. D. BUI École Polytechnique, France

J. P. CARTER University of Sydney, Australia

R. M. Christensen Stanford University, U.S.A.

G. M. L. GLADWELL University of Waterloo, Canada

D. H. HodGES Georgia Institute of Technology, U.S.A.

J. HUTCHINSON Harvard University, U.S.A.

C. HwU National Cheng Kung University, R.O. China

B. L. KariHaloo University of Wales, U.K.

Y. Y. KIM Seoul National University, Republic of Korea

Z. Mroz Academy of Science, Poland

D. PAMPlonA Universidade Católica do Rio de Janeiro, Brazil

M. B. RUBIN Technion, Haifa, Israel

A. N. SHUPIKov Ukrainian Academy of Sciences, Ukraine

T. TARNAI University Budapest, Hungary

F. Y. M. WAN University of California, Irvine, U.S.A.

P. WRIGGERS Universität Hannover, Germany

W. YANG Tsinghua University, P.R. China

F. ZIEGLER Technische Universität Wien, Austria

\section{PRODUCTION}

\section{Paulo Ney de Souza Production Manager \\ SheIla Newbery Senior Production Editor \\ SILVIO LEVY Scientific Editor}

See inside back cover or http://www.jomms.org for submission guidelines.

JoMMS (ISSN 1559-3959) is published in 10 issues a year. The subscription price for 2010 is US $\$ 500 /$ year for the electronic version, and \$660/year (+\$60 shipping outside the US) for print and electronic. Subscriptions, requests for back issues, and changes of address should be sent to Mathematical Sciences Publishers, Department of Mathematics, University of California, Berkeley, CA 94720-3840.

JoMMS peer-review and production is managed by EditFLOW ${ }^{\mathrm{TM}}$ from Mathematical Sciences Publishers.

PUBLISHED BY

mathematical sciences publishers

http://www.mathscipub.org

A NON-PROFIT CORPORATION

Typeset in LATEX

CCopyright 2010. Journal of Mechanics of Materials and Structures. All rights reserved. 


\title{
Journal of Mechanics of Materials and Structures
}

\author{
Volume 5, No. 2 February 2010
}

A critical analysis of interface constitutive models for the simulation of delamination in composites and failure of adhesive bonds

Anton Matzenmiller, Sebastian Gerlach and Mark Fiolka

185

Computational studies of collagen fibril biominerals using a virtual internal bond

model with extrinsic length scale

Ganesh Thiagarajan and Kavita Deshmukh

The simulation of stochastically excited viscoelastic systems and their stability

VADIM D. POTAPOV

Fundamental solutions for an inhomogeneous cross-anisotropic material due to horizontal and vertical plane strain line loads

Cheng-Der Wang, Jia-YAN Hou and WeI-Jer WANG

Mechanical and fracture analysis of welded pearlitic rail steels

Aldinton Allie, Heshmat A. Aglan and Mahmood Fateh

Rate dependence of indentation size effects in filled silicone rubber

Ramanjaneyulu V. S. TATIRAJU and ChUng-SOUK HAN

A novel application of a laser Doppler vibrometer in a hèalth monitoring system

DAVOOD REZAEI and FARID TAHERI

Energy absorption of a helicoidal bistable structure

Seubpong Leelavanichkul, Andrej Cherkaev, Daniel O. Adams

and FLORIAN SOLZBACHER

Decay properties of solutions of a Mindlin-type plate model for rhombic systems

Francesca Passarella, Vincenzo Tibullo and VitTorio Zampoli

A consistent refinement of first-order shear deformation theory for laminated composite and sandwich plates using improved zigzag kinematics Alexander Tessler, Marco Di Sciuva and Marco Gherlone 